\title{
Cerambyx cerdo L., ¿vulnerable o plaga?
}

\author{
Austrich, A..$^{*}$ \\ 1Universidad Autónoma de Barcelona. \\ *Autor para correspondencia: annaaustrich@gmail.com
}

\section{Resumen}

Cerambyx cerdo es un coleóptero saproxílico que vive a expensas de Quercus ilex y Quercus suber. Los signos y síntomas visibles que les ocasiona son la acumulación de serrín en la base del tronco, marchitamiento de las ramas terminales y orificios de salida de unos $20 \mathrm{~mm}$ de diámetro. Se trata de un insecto amparado por varias figuras protectoras (considerada especie vulnerable según la UICN) debido a la disminución de sus poblaciones al norte de Europa y, por lo tanto, se tienen que priorizar estrategias de conservación en estas áreas. El problema recae en la Región Mediterránea donde representa una amenaza debido a factores bióticos y abióticos, siendo la Isla de Mallorca un claro ejemplo. El objetivo del trabajo es analizar esta situación en Europa y evaluar los métodos de control más adecuados para las zonas del sur debido a su incidencia en los bosques de esclerófilas mediterráneas. A pesar de que el estado de protección en los países del norte es el adecuado, en la Región Mediterránea se tendría que rebajar el grado de protección de la especie y, como medida de control, se recomienda realizar capturas masivas mediante trampas con atrayentes específicos colocadas en troncos de gran diámetro y orientadas al sur o al oeste.

Palabras clave: cairomonas, conservación, control, Mallorca, Quercus. 


\section{Introducción}

Cerambyx cerdo L. (1758) (Col.: Cerambycidae) es un escarabajo xilófago que depende, en gran parte de su ciclo, de la madera de árboles muertos o moribundos (del género Quercus) (Buse et al., 2007; Lieutier et al., 2004).

\subsection{Morfología}

Los adultos presentan un cuerpo alargado y cilíndrico llegando hasta $55 \mathrm{~mm}$ de longitud en las hembras y $50 \mathrm{~mm}$ en los machos de una coloración castaño oscuro. Además, tienen unas antenas que llegan a sobrepasar su cuerpo (EUWSF, 2009; Lieutier et al., 2004).

En cuanto a la larvas, tienen una cabeza de color negro y un integumento blando y despigmentado con una segmentación muy marcada a lo largo del cuerpo (Lieutier et al., 2004). Al final del desarrollo larvario (5 estadios) pueden medir hasta $70 \mathrm{~mm}$ de longitud y $16 \mathrm{~mm}$ de anchura (Martín et al., 2005). Las larvas son ápodas (Lieutier et al., 2004).

Esta especie se ha confundido y citado erróneamente en diferentes puntos de España debido a su parecido morfológico con Cerambyx welensii (Viñolas y Vives, 2012). Es por esto que Sánchez (2010) argumenta que la pubescencia de los tarsos posteriores es un buen carácter para la diferenciación de estas dos especies.

\subsection{Biología}

Las hembras adultas depositan los huevos (unos trecientos como máximo) en la superficie o en grietas de la corteza de árboles muertos o moribundos durante los meses de mayo a septiembre (Sallé et al., 2014). Transcurridos unos diez días, los huevos eclosionan y salen las larvas, las cuales, durante el primer año, se encuentran en los primeros centímetros por debajo de la corteza. Al segundo año, migran hacia el interior del tronco para alimentarse del xilema. En el último estadio larvario, realizan galerías elípticas que salen al exterior formando unos orificios de hasta $20 \mathrm{~mm}$ de diámetro para facilitar la salida de los adultos (Albert et al., 2012; Lieutier et al., 2004). A continuación, las larvas dan lugar a los adultos los cuales pueden permanecer dentro de los árboles inactivos hasta que las condiciones sean favorables y salen durante los meses de mayo a septiembre (Martín et al., 2005; Núñez, 2002).

Los adultos viven entre tres y cinco semanas y se alimentan de la savia que brota de las heridas de los árboles (Quercus spp. especialmente) pero también de los nutrientes de los frutos maduros de otras especies vegetales. Se trata de un insecto crepuscular con actividad óptima entre las 18 y las 21 horas (González et al., 2013) y con una baja capacidad para dispersarse llegando tan solo a separarse del árbol donde ha nacido unos quinientos metros como máximo. El ciclo se completa entre tres y cinco años más tarde (Martín et al., 2005; Sallé et al., 2009). 


\subsection{Distribución geográfica}

Originalmente, su distribución era el norte de África y Europa siguiendo la distribución de Quercus spp., con excepción del Reino Unido (Buse et al., 2007). Según la World Conservation Monitoring Centre (1996), algunas de las zonas nativas de esta especie serían el norte de África, la región del Cáucaso, España, noreste y centro Europa, Francia, Irán, República Islámica y Turquía.

Actualmente presenta una distribución eurosiberiana en encinas y alcornoques (González et al., 2013) siendo muy común al sur de Europa pero raro y en declive al norte de su área de distribución (EUWSF, 2009). Las últimas poblaciones viables están en Francia, Alemania, Polonia y la Región Mediterránea (Buse et al., 2007).

Durante estos últimos años en la península ibérica, las superficies ocupadas por Quercus ilex L. subsp. ilex y Quercus suber L. presentan un proceso de degradación debido a una serie de factores abióticos y bióticos como la erosión, la actividad antrópica, o las enfermedades, entre otras (López et al., 2006; Sallé et al., 2014). Según Viñolas y Vives (2012), C. cerdo se encuentra en varias localidades de España siendo las zonas de la mitad oriental las que presentan una mayor densidad de población, pero con poblaciones bastante estables, exceptuando las Islas Baleares (Mallorca), donde se encuentra en expansión causando graves problemas a nivel forestal (Viñolas y Vives, 2012).

\section{4 ¿Qué árboles prefiere y qué les ocasiona?}

Los principales árboles susceptibles al ataque son las encinas (Quercus ilex) y alcornoques (Quercus suber), especialmente aquellos individuos viejos o que se encuentran en un mal estado fisiológico debido a factores ambientales o biológicos, como el déficit de agua, el ataque de otras plagas o enfermedades que les provocan una pérdida de la turgencia de las células (marchitamiento) y, por lo tanto, una reducción en la capacidad defensiva ante este xilófago (Sánchez-Osorio et al., 2005; Tur et al., 2009). Por lo tanto, C. cerdo, se considera una plaga secundaria (Sallé et al., 2014; Sánchez-Osorio et al., 2005).

Hay que tener en cuenta que no sólo puede afectar al género Quercus, sino que especies de hoja caduca como las del género Ulmus, Castanea, Betula, Salix, Ceratonia, entre otras, se pueden ver afectadas si la densidad poblacional del insecto es muy elevada (EUWSF, 2009; Sallé et al., 2014; Tur et al, 2009).

Cuando los huéspedes se ven afectados por este coleóptero inician toda una serie de mecanismos de resistencia, bioquímicos y físicos, para frenar su entrada (Wermelinger, 2004). Primero, se produce una compartimentación mediante la lignificación de las paredes celulares afectadas y la formación de un tejido interno impermeable (Sallé et al., 2014). A continuación, se desarrolla la peridermis necrótica al margen de los tejidos desarrollados posteriormente $\mathrm{y}$, si el daño es profundo, se produce la formación de un callo de cicatrización por parte del cambium. Este proceso produce la curación de la herida y la larva, al no poderse desarrollar, muere (Sallé et al., 2014). La eficacia de esta resistencia se puede ver afectada por el estado fisiológico, el genotipo y la fenología del huésped y por la actividad de la larva. Una cuestión importante es que las células adyacentes 
sintetizan y acumulan en el citosol fenoles, taninos y compuestos isoprenoides que contribuyen a la resistencia contra patógenos de la madera. No obstante, su eficacia contra insectos xilófagos aún no ha sido demostrada, (Sallé et al., 2014).

Según Albert et al., (2012) y González et al., (2013), los signos y síntomas que presentan los huéspedes afectados son:

- Acumulación de serrín en la base de los troncos.

— Orificios de salida elípticos de $20 \mathrm{~mm}$ de diámetro aproximadamente.

- Ramas secas y abscisión de hojas.

- Heridas recién hechas de una coloración rojiza por la parte interna.

\section{Objetivo}

Analizar la situación de C. cerdo en diferentes zonas de Europa ya que en determinados países se encuentra en declive (norte de Europa), mientras que en otras zonas, sobre todo en la Región Mediterránea, se está convirtiendo en un problema fitosanitario debido a la elevada densidad poblacional. A lo largo de este trabajo se analizará esta situación y se discutirán los posibles métodos de control para las zonas del sur de Europa.

\section{Cerambyx cerdo como especie vulnerable}

\subsection{Causas del declive}

Este coleóptero se encuentra en declive especialmente al norte de Europa debido a toda una serie de factores que se han producido a lo largo de los últimos cien años (Buse et al., 2007; Sallé et al., 2014; Tur et al., 2009):

- Pérdida de su hábitat natural.

- Ausencia de una continuidad de árboles susceptibles.

- Disminución de la incidencia de la radiación solar en los troncos de los árboles debido a un cierre del bosque.

Por todos estos motivos, se encuentra amparado por diferentes figuras protectoras (Lieutier et al., 2004; Sánchez-Osorio et al., 2005):

- Convenio de Berna relativo a la conservación de la vida salvaje y del medio natural en Europa. C. cerdo está citado en el Anexo II como "especie estrictamente protegida". No obstante según la legislación nacional española, las especies de invertebrados del Anexo II pasan al Anexo III como "especie protegida" (Council of Europe, 1979).

- Directiva 97/62/CEE del Consejo, de 27 de octubre de 1997, la cual es una adaptación al progreso científico y tecnológico de la Directiva Hábitats de la UE 92/43/CEE del Consejo, de 21 de mayo de 1992, relativa a la conservación de los hábitats naturales y de la flora y fauna silvestres. C. cerdo está presente en el Anexo II y IV (Council of Europe, 1979; UNIÓN EUROPEA, 2003).

- UICN la considera especie Vulnerable A1c+2c (World Conservation Monitoring Centre, 1996). 


\subsection{Estrategias de conservación}

Ante estas figuras protectoras, los Estados Miembros tienen que asegurar la conservación de C. cerdo mediante toda una serie de acciones (Davies et al., 2008; EUWSF, 2009):

- Mantener o restaurar el insecto como "estado de conservación favorable" en la Unión Europea.

- Designar espacios de la Red Natura 2000 para favorecer su estado de conservación.

- Definir estrategias de gestión forestal orientadas a maximizar la diversidad de microhábitats.

- Favorecer árboles autóctonos con varias edades con un número suficiente de ejemplares maduros y viejos con diferentes estados de senescencia.

- Aumentar la cantidad de madera muerta presente al bosque.

- Gestionar los pastos para mantener una vegetación abierta.

C. cerdo es una especie paraguas que tiene asociada una gran diversidad de especies vulnerables relacionadas con el género Quercus (Albert et al., 2012). De forma natural, actúa como regulador de los bosques atacando a árboles enfermos y débiles para dejar espacio a los individuos jóvenes con un buen estado fisiológico (Martín et al., 2005; Núñez, 2004).

\section{Cerambyx cerdo como plaga}

El problema que ocasiona $C$. cerdo es que en determinadas áreas como el sur de Francia, España e Italia (EUWSF, 2009) está causando un debilitamiento y deterioro significativo de las encinas y los alcornoques (Sallé et al., 2014). Principalmente, el aumento de las poblaciones del insecto es debido al abandono de las prácticas forestales (talas mayoritariamente) provocando un debilitamiento de los árboles que propicia su desarro1lo (Núñez, 2004).

Este insecto puede actuar como vector facilitando la entrada de hongos patógenos a través de los orificios de salida (Sánchez-Osorio et al., 2005). Uno de estos hongos que está en simbiosis con Cerambyx es Biscogniauxia mediterranea que ocasiona chancros carbonosos en encinas (González, 2013; Trapero et al., 2006).

\subsection{Factores implicados en el desarrollo de C. cerdo}

Según Trapero et al. (2006), los factores implicados en el desarrollo de C. cerdo como plaga forestal son:

- Factores de predisposición: envejecimiento de los árboles, ausencia de regeneración debido al pastoreo, malas prácticas de gestión selvícola, entre otras.

- Factores incitantes: suelen ser del tipo abiótico como las alteraciones del clima provocando un debilitamiento de las masas forestales.

— Factores contribuyentes: son de tipo biótico como las podredumbres por hongos, 
insectos perforadores de troncos, hongos causantes de chancros, entre otros, que aumentan la susceptibilidad de los árboles.

\subsection{Estrategias de gestión de C. cerdo}

Según Gallego (2013), cuando se produce un crecimiento insostenible de Cerambyx se tiene que inferir sus niveles poblacionales y sus fluctuaciones mediante trampas que capturen los individuos o estimen la densidad de las poblaciones larvales en los árboles.

Una vez se determina que el organismo se encuentra por encima del nivel de tolerancia, se tiene que elaborar un plan de actuación. Este plan prioriza las estrategias que permitan un control a largo plazo como serían las medidas preventivas y/o activas correctoras. Si no fueran suficientes, se realizarían las medidas activas. Siempre se tienen que escoger aquellas que sean menos tóxicas y agresivas para el medio ambiente, los seres humanos y la fauna y flora no diana (Plaza et al., 2009).

\subsubsection{Estrategias preventivas}

Debido a las figuras protectoras que la amparan, la selvicultura es una de las mejores estrategias para prevenir la expansión de C. cerdo (Sallé et al., 2014). Algunas de estas medidas preventivas son (Albert et al., 2012; Sallé et al., 2014; Sánchez, 2010; Trapero et al., 2006):

- Tala sanitaria o eliminación de ramas dañadas antes de la emergencia de los adultos.

— Eliminación de los árboles débiles para aumentar el vigor de los sanos (rejuvenecer).

- Promover los bosques semi-cerrados.

- Evitar las heridas en la corteza durante la extracción del corcho, sellar las heridas de poda o desinfectar las herramientas de poda.

- Quemar la madera que presente galerías y los árboles viejos muy afectados.

Una vez estas medidas preventivas se inician y, pasado un tiempo, se observa que no se obtienen los resultados esperados, se tiene que escoger la medida activa más idónea según el contexto (Plaza et al., 2009).

\subsubsection{Medidas activas}

A continuación, se citan algunas de las medidas activas que se han realizado a lo largo de estos años para controlar varias plagas forestales (algunas efectivas y otras en desarrollo) y que se podrían aplicar a $C$. cerdo.

a. Medidas físicas: las trampas de luz no son eficaces para la captura de nuestro insecto (Sánchez-Osorio et al., 2005). Estas trampas cromáticas consisten en unas placas amarillas engomadas que atraen al insecto debido a su color donde se quedan atrapados (Ribes \& Coscollá, 1992).

b. Medidas químicas: 
1. Plaguicidas repelentes e inhibidores de origen natural (vegetales y bacterianos): se tiene que profundizar más en su estudio, pero sobretodo podrían ser útiles para evitar la oviposición de algunos insectos (Sánchez, 2010).

2. Plaguicidas inorgánicos (minerales): estos productos no tienen tantos inconvenientes como los de síntesis química (son menos tóxicos) pero se tiene poco conocimiento sobre la aplicación en insectos forestales. Por lo tanto, será necesaria una mayor investigación para potenciar su utilidad (Albert et al., 2012; Sánchez, 2010).

3. Formulaciones de síntesis química: el uso de los fitosanitoarios a nivel forestal está muy limitado porque se tiene que prever los riesgos asociados que pueden ocasionar (Plaza et al., 2009; Gallego, 2013). Uno de sus principales inconvenientes es su amplio espectro de acción (Sánchez-Osorio et al., 2005). Además, según Albert et al. (2012) y Sánchez (2010), presentan una alta toxicidad y es necesario que la materia activa llegue eficazmente al organismo diana.

Se ha mostrado una efectividad del $94.85 \%$ por parte de la materia activa sistémica imidacloprid frente a Macropophora accentifer (Fam. Cerambycidae), insecto perforador de troncos en cítricos (Sánchez-Osorio et al., 2005).

c. Control biológico: Para su aplicación se requiere un estudio minucioso de todos los factores implicados y establecer los niveles de tolerancia admisibles (SánchezOsorio et al., 2005).

Se han realizado estudios con organismos entomopatógenos como el hongo Beauveria bassiana que ha resultado efectiva frente Monochamus alternatus (Cerambycidae), pero no se ha desarrollado lo suficiente para combatir C. cerdo (Albert et al., 2012; Sánchez, 2010; Sánchez-Osorio et al., 2005).

También se han realizado investigaciones con nematodos entomopatógenos, pero para C. cerdo no han sido suficientes (Albert et al., 2012; Sánchez, 2010). Las larvas, no obstante, realizan orificios de salida y se podría pensar que serían unos puntos de entrada efectivos para los nematodos. El problema recae en que estaríamos hablando de miles de hectáreas de bosque para tratar con algún producto fitosanitario que esté autorizado y el coste total del tratamiento sería elevado.

Por otra parte, también se han realizado estudios con himenópteros icneumónidos como agentes de control de plagas que atacan a los primeros estadios larvarios de $C$. cerdo. Estos artrópodos serian: Dolichomitus imperator, Ephialtes manifestator, Megarhyssa gigas, Odontocolon appendiculatus, Rhimphoctoma amoena, Ontsira longicaudis y Oobius rudnevi (Sánchez-Osorio et al., 2005).

Respecto a Oobius rudnevi (descubierto en Ucrania y al oeste de Georgia), sería el himenóptero más eficaz propuesto por Sánchez-Osorio et al. (2005). Durante unos 10-15 minutos, este parasitoide libera sus huevos al interior de los de $C$. cerdo donde se desarrollan (Gumovsky et al., 2013; Trjapitzin et al., 1963; Zhang et al., 2005). Se ha observado que se produce una sincronización entre estas dos especies, ya que los huevos de Cerambyx y las hembras de los himenópteros coinciden en el tiempo (mayo principalmente) (Gumovsky et al., 2013).

La estrategia de control biológico se encuentra aún poco desarrollada para especies 
perforadoras de madera de Europa asociadas a Quercus y la literatura sobre los enemigos naturales es bastante limitada y poco referenciada (Albert et al., 2012; Gallego, 2013; Sánchez, 2010).

d. Medidas bioracionales: serían métodos de captura masiva utilizando trampas cebadas con sustancias atrayentes (Sánchez, 2010).

Lo ideal sería un método de atracción específica para la especie de interés, ya sea para capturar, repeler o confundir en el momento más adecuado para asegurar una alta selectividad del método (Albert et al., 2012; Sánchez, 2010).

Para llevarlo a cabo, es imprescindible un estudio minucioso de los parámetros relacionados con la localización de los árboles susceptibles al ataque mediante el estudio de los semioquímicos. La electrofisiología de estímulos olfativos y, en particular, la técnica de la electroantenografía (EAG), proporciona una valiosa información para el estudio del comportamiento olfativo que permite a los insectos la identificación de los compuestos volátiles de las plantas para localizar sus huéspedes y/o detectar las feromonas para localizar sus congéneres (Sánchez, 2010).

Primero de todo, se tiene que determinar cuáles son los compuestos orgánicos volátiles (COV) que emiten los árboles (cairomonas), sobretodo los Quercus, porque son los principales árboles atacados por C. cerdo. De esta forma, se podrán desarrollar unos semioquímicos mucho más específicos para controlar sus poblaciones (Albert et al., 2012; Sánchez, 2010; Sánchez-Osorio et al., 2005; Sánchez-Osorio et al., 2006).

La emisión de los COV viene determinada genéticamente, de tal forma que cada especie produce sus propios COV (alta especificidad). Se ha observado que durante el día y los meses de primavera y verano la producción de COV se incrementa debido a la evolución en paralelo con la transpiración foliar (Sánchez, 2010). Esta producción se podría relacionar con la actividad de los adultos de C. cerdo.

Algunos de los COV que emiten Quercus ilex y Q. suber serían los ácidos orgánicos (ácido fórmico y ácido acético) y los compuestos C6-C10 no terpenos (en encina se ha determinado 3hexeno-1-ol, o-ectoxietilo acetato y fenol). Pero a los que se les ha dado más importancia son los monoterpenos. En la Tab. 1 se puede observar el porcentaje de los principales monoterpenos que emiten tanto la encina como el alcornoque (Sánchez, 2010).

Tabla 1. Porcentaje de monoterpenos respecto el total de COV en $Q$. ilex y $Q$. suber. (Tabla modificada de Sánchez, 2010).

\begin{tabular}{lccccc}
\hline Especie & Alfa-pineno & Beta-pineno & Limoneno & Sabineno & Mirceno \\
\hline Quercus ilex & $29-37 \%$ & $21-24 \%$ & $11-27 \%$ & $11-21 \%$ & $6-14 \%$ \\
Quercus suber & $24-28 \%$ & $14-18 \%$ & $23-33 \%$ & $23-26 \%$ & $3-5 \%$ \\
\hline
\end{tabular}

Según Sánchez (2010), el 80-90\% de las sustancias emitidas por las hojas y cortezas de los árboles que atraen a C. cerdo son: alfa-pineno, beta-pineno, limoneno, sabineno y mirceno.

Se tendría que determinar con más exactitud cuáles son los COV que atraen a los 
individuos de C. cerdo para diseñar unos atrayentes con una alta especificidad. La Fig. 1 nos muestra la emisión de diferentes monoterpenos para especies idóneas $(Q$. ilex), para especies que podrían ser idóneas si hay una densidad de población del coleóptero elevada (Ceratonia siliqua y Salix sp.) y para especies no diana (Pinus halepensis y Quercus coccifera) (Datos extraídos de Owen et al., 2001).

En principio, los árboles diana para esta especie tendrían que emitir los mismos COV

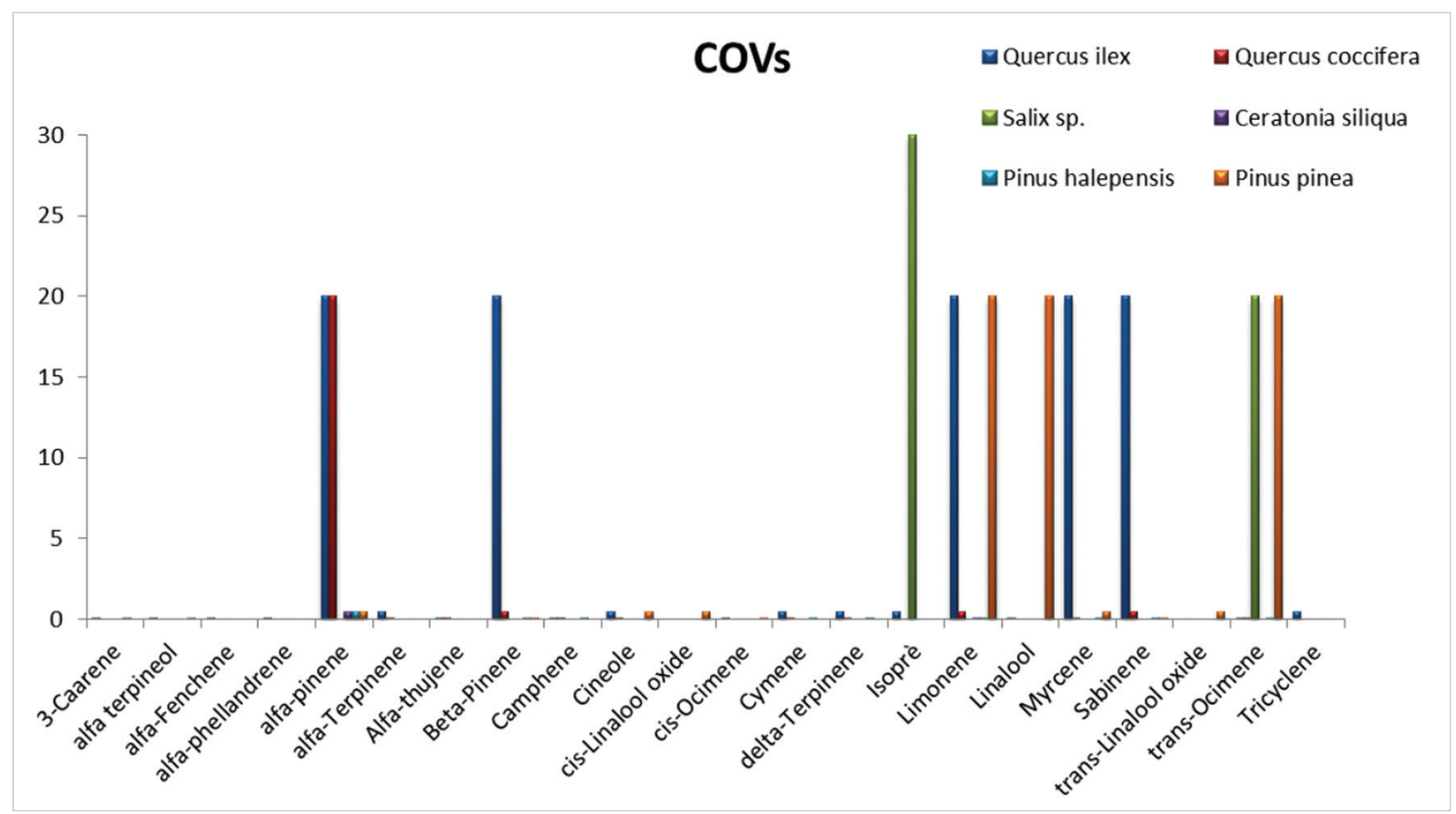

Figura 1. Representación de los compuestos orgánicos volátiles emitidos por diferentes especies vegetales. Los valores son aproximaciones al alza (Owen et al., 2001).

o con pequeñas variaciones. Pero tal y como se puede observar en el artículo de Owen et al. (2001), los resultados no son los esperados. No obstante, los monoterpenos que emite la encina son bastante diferentes tanto en tipo como en cantidad en comparación a los árboles no diana, lo cual podría confirmar las preferencias que tiene C. cerdo.

Además, ECONEX (1986), recientemente ha desarrollado el atrayente ECONEX CERAMBYX específico para este coleóptero. Consiste en una solución líquida compuesta por: $70 \%$ etanol, $0.19 \%$ acetato de etilo, $0.12 \%$ de 3 -metilo- 1 -butanol, $0.1 \%$ acetaldehido, 0.05\% 2-metilo-1-propanol, 0.04\% 2-metilo-1-butanol i 29.5\% de agua destilada. (Patente ES 2370 221B1, Universidad de Huelva, España).

Si hablamos de la trampa, Econex S.L. ha puesto a la venta CROSSTRAP. Es una trampa forestal de intercepción del vuelo para la captura de insectos forestales. Está formado por unas láminas donde el insecto choca y luego precipita a un embudo que lo dirige a un colector desmontable situado al final de la trampa quedándose los insectos atrapados (ECONEX, 1986).

Además, el lugar de localización de las trampas en los árboles es crucial para la cap- 
tura de los adultos. Según los argumentos que propone Albert et al. (2012) en su artículo sobre la localización de los orificios de salida de los individuos adultos, las trampas se podrían colocar en árboles de gran diámetro (generalmente de más edad y con variedad de microhábitats) y en las partes orientadas al sur y/u oeste (la radiación aumenta el calor en estas partes ayudando al desarrollo de la larva durante los meses de invierno).

\section{Conclusiones}

El estado de protección de la especie en las zonas donde se considera una amenaza para la supervivencia de los bosques de Quercus spp. se debería reconsiderar por parte de las administraciones. Si no se pudiera modificar el marco legal de protección que la ampara, las actuaciones deberían ir encaminadas a realizar capturas masivas de forma controlada y puntual en zonas gravemente afectadas. No parece coherente que este coleóptero que está considerado "especie de interés comunitario" por la Directiva Hábitats junto con los bosques de esclerófilas mediterráneas donde habita (especie dominante es Q. ilex, juntamente con $Q$. rotundifolia), tengan el mismo estado de protección cuando $C$. cerdo es una de las principales amenazas para estos bosques en la Región Mediterránea (González et al., 2013).

La isla de Mallorca es un ejemplo de la grave problemática que está generando este coleóptero a sus bosques de encinas las cuales están severamente afectadas donde el número de individuos de $C$. cerdo está aumentando año tras año (Núñez, 2004). Desde los años 70 hasta hoy, su población ha incrementado exponencialmente en toda la geografía mallorquina, atribuyéndose prácticamente todos los daños causados en las masas forestales del género Quercus de la isla a la acción de este coleóptero (González et al., 2010; Núñez, 2004).

Una alteración de las condiciones climáticas, la densidad de especies invasoras y patógenos, juntamente con otros factores como el cambio de uso, propician que especies secundarias como $C$. cerdo que no son más que especies oportunistas que aprovechan la abundancia de sus huéspedes proliferen (Martín et al., 2005). Además, estos factores producen una alteración en las emisiones de los COV y, por lo tanto, una alteración para las poblaciones de C. cerdo el cual se guía por estos compuestos (Martín et al., 2005; Sallé et al., 2014; Sánchez, 2010).

En respuesta a la pregunta “Cerambyx cerdo, ¿vulnerable o plaga?”, durante todo el trabajo se ha intentado exponer la problemática que lleva asociada y que se resume en que dependiendo de la región, se considera una especie amenazada o perjudicial para el medio ambiente. La respuesta depende de la zona en que hablemos. Las zonas del sur de Europa, donde sus poblaciones se han incrementado, se deberían priorizar las estrategias selvícolas para disminuir la densidad de las poblaciones; mientras que en las zonas del norte se tendrían que establecer toda una seria de medidas para potenciar el desarrollo de la especie y favorecer poblaciones estables a largo plazo.

Últimamente, se han desarrollado estudios para evaluar el daño ocasionado en los bosques de Mallorca mediante los muestreos in situ con búsqueda directa de los individuos de $C$. cerdo para conocer los niveles poblacionales para cada zona analizada 
(González et al., 2010; Núñez, 2003). Sin embargo, hacen falta más estudios sobre el comportamiento del insecto, de los niveles poblacionales asociados a situaciones de daños graves en árboles, la capacidad de dispersión o la forma de seleccionar sus huéspedes (Sánchez-Osorio et al., 2005).

\section{Agradecimientos}

Primero de todo agradecer a mi tutor de la universidad, Fernando García del Pino, por la ayuda y apoyo durante todo el desarrollo del trabajo. También tengo que agradecer a Josep María Riba (Doctor Biólogo-Fitopatólogo) y a Luís Núñez (Cap de Servei de Sanitat Forestal, Islas Baleares), expertos en Cerambyx cerdo, por las respuestas a las dudas planteadas. Y finamente, a toda mi familia por el apoyo recibido.

\section{Bibliografía}

Albert, J., Platek, M., Cizek, L., 2012. Vertical stratification and microhabitat selection by the Great Capricorn Beetle (Cerambyx cerdo) (Coleoptera: Cerambycidae) in open grown, veteran oaks. European Journal of Entomology. 109 (4), 553-559.

Buse, J., Schröder, B., Assmann, T., 2007. Modelling habitat and spatial distribution of an endangered longhorn beetle - A case study for saproxylic insect conservation. Biological conservation. 137 (3), 372-381. doi:10.1016/j.biocon.2007.02.025.

Council of Europe, 1979. Convention on the Conservation of European Wildlife and Natural Habitats. Environmental Conservation, 7(02), 143. doi: 10.1017/S0376892900007189

Davies, Z., Tyler, C., Stewart, G., Pullin, A., 2008. Are current management recommendations for saproxylic invertebrates effective? A systematic review. Biodiversity Conservation. 17 (1), 209-234. doi: 10.1007/s10531-007-9242-y.

ECONEX S.L. 1986. Crosstrap. Econex sanidad agrícola, especialistas en feromonas y trampas. http://www.e-econex.com/trampas-para-insectos/. 15 Abril 2015.

EU Wildlife and Sustaintable Farming project (EUWSF)., 2009. Great Capricorns beetle Cerambyx cerdo factsheet. Habitats Directive, Annex II \& IV. Office. http://ec.europa. eu/environment/nature/natura2000/management/docs/Cerambyx $\% 20$ cerdo\%20factsheet\%20-\%20SWIFI.pdf. 21 Octubre 2014.

Gallego, D., 2013. El uso de feromonas y cairomonas en el control de plagas forestales y del jardín. III Jornadas Internacionales sobre feromonas, atrayentes, trampas y control biológico.

González, E., Gallego, D., Lencina, J.L., Closa, S., Muntaner, A., Núñez, L., 2010. Propuesta de una metodología para la determinación de los niveles de infestación por Cerambyx cerdo (Linnaeus, 1758) (Coleoptera: Cerambycidae). Evaluación de los niveles de infestación en Mallorca, año 2009. Boletín Sanidad Vegetal Plagas. 2, 157-163.

González, E., Núñez, L., Lencina, J.L., Gallego, D., 2013. Evaluación de los niveles de infestación y medidas de control de Cerambyx cerdo (Linnaeus, 1758) en Mallorca (Islas Baleares). Congresos forestales.

Gumovsky, A.V., Simutnik, S.A., Prokhorov, A.V., Tymobcknn, A.B., Cnmythnk, C.A., 
Mpoxopob, A.B., 2013. Life-history review of Oobius zahaikevitsbi Trjapitzin, 1963 (Hymenoptera: Encyrtidae), an egg parasitoid of jewel beetles (Coleoptera: Buprestidae) Russian Entomological Journal. 22(3), 181-188.

Lieutier, F., Day, K.R., Battisti, A., Grégorie, C.J., Evans, H.F., 2004. Bark and wood boring insects in living trees in Europe, a synthesis. Kluwer, Dordrecht, 445-498. doi:10.1007/978-1-4020-2241-8.

López, G., Sánchez-Osorio, I., Domínguez, L., 2006. Cerambicidos xilófagos de encinas y alcornoques: estudio bioecológico y control de poblaciones. Bol. Inf. CIDEU. 1, 39-144.

Martín, J., Cabezas, J., Buyolo, T., Patón, D., 2005. The relationship between Cerambyx spp. damage and subsequent Biscogniauxia mediterranum infection on Quercus suber forests. Forest Ecology and Management. 216 (1-3), 166-174. doi:10.1016/j.foreco.2005.05.027.

Núñez, L., 2002. El banyarriquer. L'insecte perforador que ataca als alzinars. Quadern de natura núm.14, Conselleria de Medi Ambient, Govern de les Illes Balears.

Núñez, L., 2003. El banyarriquer a les Illes Balears I. L'Observador del Pla de Mallorca núm. 12: 26.

http://www.caib.es/sacmicrofront/contenido.do?mkey=M149\&lang=ES\&cont=3423. 25 Noviembre 2014.

Núñez, L., 2004. El caso del gran capricornio o banyarriquer (Cerambyx cerdo Linnaeus, 1758) en las Islas Baleares. Revista FORESTA n²4. Del Colegio de Ingenieros Técnicos Forestales. $4^{\circ}$ trimestre de 2004.

Owen, S. M, Boissard, C., Hewitt, C.N., 2001. Volatile organic compounds (VOCs) emitted from 40 Mediterranean plant species: VOC speciation and extrapolation to habitat scale. Atmospheric Environment. 35 (32), 5393-5409. doi: 10.1016/S1352-2310(01)00302-8.

Plaza, T., Pando, P., Albiñana A. 2009. Curs de nivell qualificat de manipulador i aplicador de productes fitosanitaris. Departament d'Agricultura, Alimentació I Acció Rural, Barcelona.

Ribes, A., Coscollá, R., 1992. Notas sobre el seguimiento poblacional de Frankliniella occidentalis Perg. en el cultivo del fresón. Bolentín de Sanidad Vegetal Plagas. 18, 569-584.

Sallé, A., Nageleisen, L.M., Lieutier, F., 2014. Bark and wood boring insects involved in oak declines in Europe: Current knowledge and future prospects in a context of climate change. Forest Ecology and Management. 328, 79-93. doi: 10.1016/j.foreco.2014.05.027.

Sánchez, I., 2010. Orientación olfativa de "Cerambyx welensii" Küster y "Prinobius germani" Dejean, principales cerambícidos xilófagos de encina ("Quercus ilex L. subsp ballota) y alcornoque ("Quercus suber L.") para la localización de hospedantes. Tesis Doctoral. Universidad de Huelva.

Sánchez-Osorio, I., Tapias, R., Domínguez, L., López, G., 2005. Cerambícidos xilófagos de encina y alcornoque en Andalucía: algunas notas sobre la identificación de especies del "grupo Cerambyx", sus daños al arbolado y las posibilidades de control de sus poblaciones. Actas del IV Congreso Forestal Español.

Sánchez-Osorio, I., Tapias, R., López, G., Domíguez, L., 2006. Estructura básica y principales parámetros de un dispositivo para el estudio electroantenográfico de estímulos olfativos en cerambícidos. Boletín Sanidad Vegetal Plagas. 32, 109-119.

Trapero, A., Romero, M.A., Sánchez, J.E., Jiménez, J.J., Sánchez, M.E., 2006. La seca de encinas y alcornoques en Andalucía: decaimiento y enfermedad. Bol. Inf. CIDEU. 1, 7-14.

Trjapitzin, V.A., 1963. Species of the genus Oobius gen.n. (Hymenoptera, Encyrtidae) in the USSR. Acta Entomologica Musei Nationalis Pragae. 35, 543-547.

Tur, C., Núñez, L., Closa, S., Blasco, I., Muntaner, A., Alemany, A., 2009. Estudio preliminar 
sobre métodos de control de la población de Cerambyx cerdo (Coleoptera: Cerambycidae) en los encinares de Mallorca. Congresos Forestales. doi: 10.1017/CBO9781107415324.004.

UNIÓN EUROPEA., 2003. Directiva 92/43/CEE del Consejo, de 21 de mayo de 1992, relativa a la conservación de los hábitats naturales y de la fauna y flora silvestres. http://www.mediterranea.org/cae/direct_92_43_cee.htm. 27 Diciembre 2014.

Viñolas, A., Vives, E., 2012. Cerambyx cerdo. En; W.AA., Bases ecológicas preliminares para la conservación de las especies de interés comunitario en España: Invertebrados. Ministerio de Agricultura, Alimentación y Medio Ambiente. Madrid. 63 pp.

Wermelinger, B., 2004. Ecology and management of the spruce bark beetle Ips typographus - a review of recent research. Forest Ecology and Management. 202 (1-3), 67-82. doi: 10.1016/j.foreco.2004.07.018.

World Conservation Monitoring Centre, 1996. Cerambyx cerdo. The IUCN Red List of Threatened Species 1996.

http://dx.doi.org/10.2305/IUCN.UK.1996.RLTS.T4166A10503380.en. 27 Abril 2016.

Zhang, Y.Z., Huang, D.H., Zhao, T.H., Liu, H.P., Bauer, L.S., 2005. Two new species of egg parasitoids (Hymenoptera: Encyrtidae) of wood-boring beetle pests from China. Phytoparasitica. 33(3), 253-260. doi: 10.1007/BF02979863. 
